University of South Florida

DIGITAL COMMONS

Digital Commons @ University of

@ UNIVERSITY OF SOUTH FLORIDA

South Florida

History Faculty Publications

History

2011

\title{
Automatic Classification of Decorative Patterns in the Minoan Pottery of Kamares Style
}

\author{
Filippo Stanco \\ University of Catania \\ Davide Tanasi \\ Arcadia University, dtanasi@usf.edu \\ Giuseppe C. Guarnera \\ University of Catania \\ Giovanni Gallo \\ University of Catania
}

Follow this and additional works at: https://digitalcommons.usf.edu/hty_facpub

\section{Scholar Commons Citation}

Stanco, Filippo; Tanasi, Davide; Guarnera, Giuseppe C.; and Gallo, Giovanni, "Automatic Classification of Decorative Patterns in the Minoan Pottery of Kamares Style" (2011). History Faculty Publications. 67. https://digitalcommons.usf.edu/hty_facpub/67

This Book Chapter is brought to you for free and open access by the History at Digital Commons @ University of South Florida. It has been accepted for inclusion in History Faculty Publications by an authorized administrator of Digital Commons @ University of South Florida. For more information, please contact digitalcommons@usf.edu. 


\section{Pattern Recognition and Signal Processing in Archaeometry:}

Mathematical and Computational Solutions for Archaeology

Constantin Papaodysseus

National Technical University of Athens, Greece 
Managing Director:

Senior Editorial Director:

Book Production Manager:

Development Manager:

Development Editor:

Acquisitions Editor:

Typesetters:

Print Coordinator:

Cover Design:
Lindsay Johnston

Heather Probst

Sean Woznicki

Joel Gamon

Myla Harty

Erika Gallagher

Jennifer Romanchak

Jamie Snavely

Nick Newcomer, Greg Snader

Published in the United States of America by

Information Science Reference (an imprint of IGI Global)

701 E. Chocolate Avenue

Hershey PA 17033

Tel: 717-533-8845

Fax: 717-533-8661

E-mail: cust@igi-global.com

Web site: http://www.igi-global.com

Copyright (C) 2012 by IGI Global. All rights reserved. No part of this publication may be reproduced, stored or distributed in any form or by any means, electronic or mechanical, including photocopying, without written permission from the publisher.

Product or company names used in this set are for identification purposes only. Inclusion of the names of the products or companies does not indicate a claim of ownership by IGI Global of the trademark or registered trademark.

\section{Library of Congress Cataloging-in-Publication Data}

Pattern recognition and signal processing in archaeometry : mathematical and computational solutions for archaeology / Constantin Papaodysseus, editor

$$
\text { p. ; cm. }
$$

Summary: "This book offers state of the art research in computational pattern recognition and digital archaeometry, showing readers how to discover reliable automated methods for quickly reconstructing archaeological materials and benefit from the application of non-destructive, automated processing of archaeological finds"--Provided by publisher.

Includes bibliographical references and index.

ISBN 978-1-60960-786-9 (hardcover : alk. paper) -- ISBN 978-1-60960-787-6 (ebook) -- ISBN 978-1-60960-788-3 (print \& perpetual access) 1. Archaeometry. 2. Archaeology--Data processing. 3. Archaeology--Computer simulation. 4. Pattern recognition systems. 5. Imaging systems in archaeology. I. Papaodysseus, Constantin, 1954- II. Title.

CC75.7.P38 2011

930.10285--dc23

$$
2011041829
$$

British Cataloguing in Publication Data

A Cataloguing in Publication record for this book is available from the British Library.

All work contributed to this book is new, previously-unpublished material. The views expressed in this book are those of the authors, but not necessarily of the publisher. 


\title{
Chapter 6
}

\section{Automatic Classification of Decorative Patterns in the Minoan Pottery of Kamares Style}

\author{
Filippo Stanco \\ University of Catania, Italy \\ Davide Tanasi \\ Arcadia University, USA
}

\author{
Giuseppe Claudio Guarnera \\ University of Catania, Italy \\ Giovanni Gallo \\ University of Catania, Italy
}

\begin{abstract}
An important feature of the Minoan culture is the pottery of Kamares style, that documents the Cretan cultural production between the first half of the $2^{\text {nd }}$ millennium $B C$. This high level painted production, characterized by the combination of several diverse motifs, presents an enormous decorative repertoire. The extraordinary variety of combinations between elementary motifs according to a complex visual syntax makes interesting the automatic identification of the motifs, particularly upon potsherds. A complete pipeline to accomplish this task is still a challenge to Computer Vision and Pattern Recognition. Starting from a digital image ROI identification, motif extraction, robust contour detection should be performed to obtain a bag of digital shapes. In a second phase each of the extracted shapes has to be classified according to prototypes in a database produced by an expert. The co-occurrence of the different shapes in a specimen will, in turn, be used to help the archaeologists in the cultural and even chronological setting.
\end{abstract}




\section{INTRODUCTION}

In the past decades, the application of the computer science in the archaeological research, and especially in the field of prehistory, turned out from a simple auxiliary technology into a cognitive strategy influencing the approach to the ancient artifacts. Although the introduction of the database enhanced the possibility of dealing with large amount of text data, the problem of taking into account huge groups of visual data still remains unsolved. One common case that makes hard the initial steps of an archaeological study is for example the analysis of decorative repertoires of some prehistoric pottery classes that are characterized by the exuberant use of a multiplicity of motifs. The most complicated artistic production of the Mediterranean prehistory is certainly the Kamares style pottery (Figure 1), flourished in Crete in the first half of the $2^{\text {nd }}$ millennium, which main feature is to present a complex system of polychrome painted decoration with a rich decorative alphabet aimed to produce an endless variation of visual results. The highest number of vessels and potsherds available of this class all over Cretan territory and the fact that a complete framework for the Kamares decorative grammar and the associations 'motif/ves$\mathrm{sel} /$ site/chronological layer' is far from being understood, determined a stop in the cognitive progress of the Minoan civilization.

In this perspective Computer Vision and Pattern Recognition could provide a great support in automatically assisting the archaeologists in classification of Kamares pottery fragments (Figure 2), especially considering that in many cases, the visual informations available are of several kinds, like watercolors, black and white

Figure 1. Examples of Kamares style vessels (this image has been obtained as a collage from several public sources)
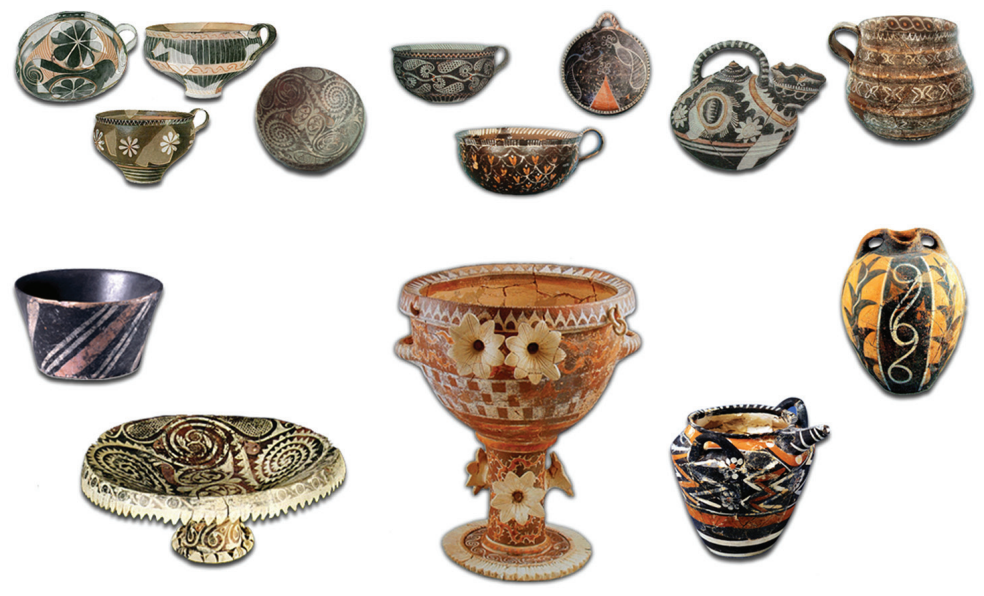
and color photographs of unequal resolution and quality, pencil sketches and high quality digital photographs. Within the research program Archeomatica Project (IPLab, 2010) of the Catania University, devoted to the application of Computer Graphics and Image Processing techniques in the field of prehistoric archaeology (Stanco, Battiato, Gallo, 2011), a specific study for the developing of an automatic classification system of the Kamares simple decoration elements (Farinella, Stanco, Tanasi, 2008), (Guarnera, Stanco, Tanasi, Gallo, 2010) (Gallo, Stanco, Tanasi, in press) started under the inspiration of the Center of Cretan Archaeology (2010) of the Catania University, dealing with the Kamares pottery from Phaistos (Levi, Carinci, 1988), (Carinci, 1997).

The application of this research strategy on the available visual corpus of image data is the object of the research that we present in this chapter. The chapter is structured as follow: in Sections 1.2 we introduce the Kamares pottery. Section 1.3 shows the proposed technique. In Section 1.4 a review of existing shape similarity techniques

\section{Background: The Kamares Pottery}

The Kamares style pottery represents the main artistic feature of the Minoan civilization between the 20th and 17th century BC and its technical and stylistic level achieved was never equaled in the Aegean Bronze Age (Walberg, 1978), (Walberg, 1983), (Walberg, 1987), (Walberg, 2001), (Knappett, 1999), (Zois, 1998). This class, with a large lifespan that covers the Protopalatial and Neopalatial periods, can be divided in four main phases related to the local chronology arranged by A. Evans (1921): Pre-Kamares or 1 (Middle Minoan IA), Early Kamares or 2 (Middle Minoan

Figure 2. Selection of Kamares pottery sherds from Phaistos (courtesy of Prof. V. La Rosa)

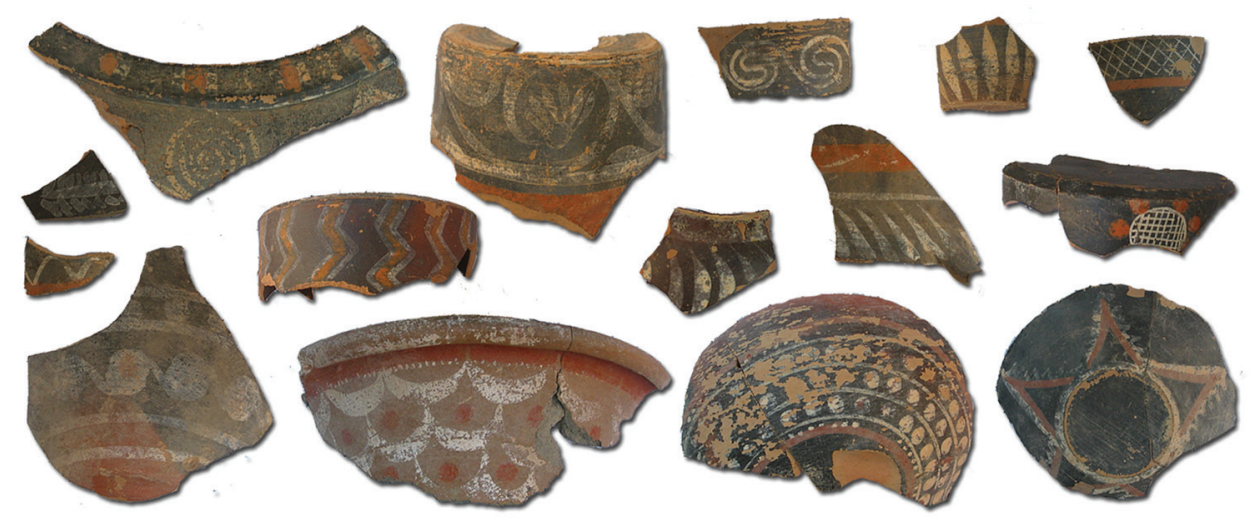


IB/IIA), Classical Kamares or 3 (Middle Minoan IIA/IIB/IIIA), Post-Kamares or 4 (Middle Minoan IIIA/IIIB). Kamares ware is named for finds first identified at the end of 19th century in the Kamares cave sanctuary in Mount Ida, and its diffusion on the Cretan territory can be distinguished between the elegant production of the palatial centers of Knossos and Phaistos (Palatial Kamares) (Day, Wilson, 1998) and the less impressive creations of the provincial workshops of Eastern, Western, Central and Eastern-Central Crete (Provincial Kamares areas 1-4) as in Figure 3.

Fine fabric, thrown on the wheel, sometimes with very thin bodies (2-3 mm like in the egg shell varieties), painted with polychrome pigments on dark background, the Kamares style has a vast assemblage of shapes and its distinctive feature is a huge repertoire of exuberant decorative motifs often resembling naturalistic atmospheres (Figure 1). About the colors, the black slip which covers most of Kamares vessels has been identified as a clayish paint layer containing iron oxides of spinel type with a high content of potassium. It is produced by exposure to high temperatures in a reducing atmosphere, thereby turning in into a black sintered layer. The red color consists of red ochre, which in contrast with the black slip does not contain potassium at all. As a result of the lower content of potassium, the red paint layer does not melt and turn into a sintered layer in a reducing atmosphere but it remains

Figure 3. Map of Crete showing principal sites with Kamares style pottery (Walberg, 1983)

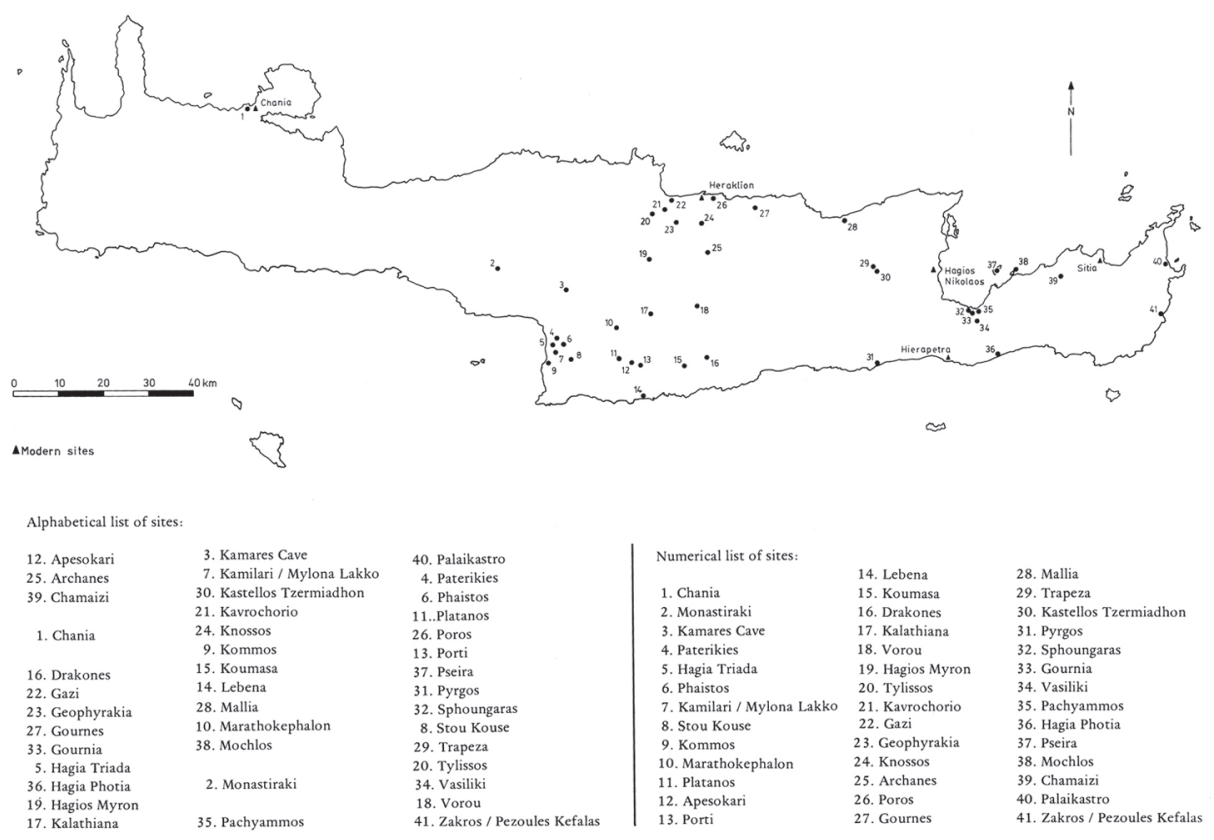


porous and turns bright red when exposed to oxidation. The white pigment is composed by calcium silicate or talc depending on the chronological period of the production and of the production centre itself. In the decoration of large vessels of domestic type also a less elaborated and much lasting dark on light decoration system is used as it was found appropriate for vases which had to be strong and practical rather than attractive.

In a preliminary survey, seventy-four different shapes were identified including both the decorated and fine ware and domestic and specialized forms. During the four chronological phases, open and closed shapes are equally preferred and even if there is a general conservativeness in the use of the same formal typologies some specific tendencies for each phase can be defined (Figure 4).

For what concerns the decoration, the Kamares style is basically characterized by the application of thirty-one different core motifs (abstract, rectilinear, pictorial, pictorialized motifs) and patterns (stone, rock, sponge and metal surface patterns), each of them with several levels of varieties, composed together in order to obtain complex decorative syntaxes joined to create much more complicated composite designs.

In general the repertoire of core motifs maintains itself the same in both the main production districts, the Palatial and Provincial, and basically remains unaltered during all the chronological development, just with few elements peculiar for some phases. But it must be also considered that a core motif, even simple, can deeply change shape when transformed from a $2 \mathrm{D}$ sketch to a $3 \mathrm{D}$ design on the vessel body, when combined with copies of the same motif or joined with other motifs,

Figure 4. Schematic shape taxonomy of the Kamares pottery repertoire (Walberg, 1978)

\begin{tabular}{|l|l|l|l|l|}
\hline $\begin{array}{l}\text { Pre- } \\
\text { Kamares }\end{array}$ & & Open & Tall, elongated \\
\hline $\begin{array}{l}\text { Early } \\
\text { Kamares }\end{array}$ & & & & \\
\hline $\begin{array}{l}\text { Classical } \\
\text { Kamares }\end{array}$
\end{tabular}


Figure 5. Schematic taxonomy and possible elaborations of some Kamares decorative motifs (Walberg, 1978)

\begin{tabular}{|c|c|c|c|c|c|c|c|c|c|c|c|}
\hline \multicolumn{7}{|c|}{$J$-spirals } & \multicolumn{4}{|c|}{ C-spirals S-spirals Spiral derivatives } & \multirow{2}{*}{ Disc-spirats } \\
\hline Single & & & & & & 6 & 0 & $\infty$ & யயIIII & & \\
\hline Combined & coumating & & 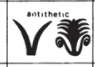 & D० & 60 & ๘ొฮี & $D$ & 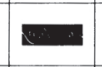 & $<$ & स्य & \\
\hline Elaborate & & & 籍 & ॠळ & & (a) & 势 & (a) & 5 & & 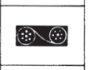 \\
\hline Pictorialized & gom & \pm & 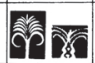 & & 酷 & & & & & & \\
\hline Pictorial & & 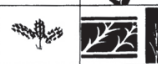 & 沙 & & i & כ经 & & & 5 & & (c) \\
\hline
\end{tabular}

when elaborated or pictorialized and in relation to different chronological phases. As a result all this makes very hard, in some cases, the classification of each element (Figure 5).

About the composition of decorative elements and its relation with the tectonic of the vessel, two different method can be identified. First is the unity decoration that depends on the shape of the vase and that can be circumcurrent, that means planned with regard to the whole vessel body and composed by encircling zones, facial, that means planned with regard only to one part of the vessel body as seen from a special angle, and zonal, that means composed by a system of decorated zones related to each other in order to create coherent designs. Second is the structural decoration that consists in separation of the vase into different parts and accentuates the horizontal and vertical axes. In the both type of decoration a field division for the location of the motifs is applied, selecting different parts of the body vessels for the definition of the limits of the decorated area. In the field division the choice of the decoration strategy for the accessory parts of the vase, like rim, lip, handle, spout, is very peculiar of each period and production centre. It must be also considered that both the shapes and the decoration contain dynamic effects and the decoration cannot merely be described as a movement across a surface, as in the case of a static background. It must be described as a movement in the same direction or opposed to the movement inherent in the vessel shape. The dynamic effects of the vase and the decoration together create the overall effect, that can give, for example, the illusion of the contraction or expansion of some vessel parts. In the selection of the composition strategies it's possible to find difference between the Palatial and Provincial production and to define tendencies from the Pre-Kamares to the Post-Kamares phases (Macgillivray, 1998).

The overall effect of a Kamares vessel is sometimes completed by the application of an accessory decoration represented by incision, relief and applied plastic details or by the use of complementary embellishment techniques like the ripple, barbotine 
and the trickle decoration. Finally, it must be noticed that differences can be find also within the same centre and in the same period because, besides the guidelines of this production, the essence of the style itself was strongly influenced by the creativity and aesthetic concept of the single artisan, that constantly elaborated the core motifs and experimented new ways of expression. As a result, the Kamares style is more than the simple combination of syntaxes of elementary motifs with different vessel type. It is a complicated interplay, carried out to the smallest detail, where every elements has an important part, especially in the Palatial production. The fact that each Kamares style product depends by many quantifiable variables (time, place, vessel shape, high number of probable composition of core decorative elements, colors and accessory decoration techniques) and just by one variable not quantifiable at all that is the unpredictable will of the artisan, it made quite impossible for the archaeologists specialized in the Middle Minoan pottery to carry out a exhaustive study of the main features of this style, also because of the huge amount of data represented by millions of decorated potsherds.

The first study of classification of the Kamares pottery was carried out by G. Walberg in 1978 and it was dedicated to the Palatial production of Phaistos and Knossos (Walberg, 1978), (Walberg, 1987) (Figure 3). In this work, the role of Phaistos, as the most important production centre and also the only site with a clear chronological progression in the development of the production itself, was pointed out and a corpus of core motifs and patterns found there was published. Furthermore, the features characterizing the climax of the Kamares style were find between Middle Minoan IIA and Middle Minoan IIIA, within the phase named Classical Kamares. In 1983 a second reassessment of the Kamares materials from Provincial districts was carried out by the same author (Walberg, 1983), emphasizing analogies and differences between Palatial and Provincial production and trying to rebuild the decorative repertoire, as whole as possible, in order to enrich the main assemblage and obtain a complete study and classification of the Kamares pottery.

After the Walberg's corpora, a large quantity of new Kamares vessels were found and published together with groups of pottery coming from old excavations, in both cases often fragmented potsherds. This re-opened the problem for the archaeologists of dealing with a class of materials so variable present in huge amount of specimens. In particular the interpretation of the decorative motifs and syntaxes partially preserved on the sherds, in order to ascribe them to the repertoire of a specific workshop, trying to match the fragmentary information with a standard collection of visual references arranged by the scholars, has become the hardest part of the research.

Although the goal of an exhaustive classification of Kamares pottery's production is likely to be unobtainable with the present state of the art, Computer Vision and Pattern Recognition could provide a great support in automatically assisting 
the archaeologists in the classification task. Our contribution illustrates a complete pipeline to automatically process these data.

The processing starts with the extractions of a clean representation of the decorative designs. Our goal is to create a standardized database of "shapes" that could be successively automatically investigated with Pattern Recognition methods.

\section{Iconographical Documentation Available}

Besides the large amount of specimens available, another significant problem in an exhaustive study of the Kamares style pottery is dealing with an heterogeneous group of iconographical data. For the two most important Palatial production centers, Phaistos and Knossos, the documentation is basically composed by watercolors, black and white pictures, technical drawings in scale with front view and section and reconstructive unscaled perspective drawings. The best preserved vessels representing the highest stylistic level of the Kamares style, in the editions of the two sites, were illustrated by unscaled watercolors carried out by E. Stafani, R. Oliva and Th. Fanourakis for Phaistos and D. Mackenzie for Knossos. In the reproduction of the complex Kamares painted decoration, each modern artist or technician at work was influenced by his own personal taste and this caused, in many cases, alteration and distortion of the original shape of the motifs and of the original scheme of the syntaxes.

It must be also considered that the draftsmen working in Crete in the first half of the XX century, had different formation and artistic education. For the reproduction of the Phaestian Kamares, E. Stefani, chosen by the director L. Pernier for the documentation of the excavation, was an architect. Decades later, D. Levi, new head of the expedition, chose R. Oliva and Th. Fanourakis, two painters strongly influenced by his archaeological point of view. Otherwise at Knossos, a large part of the graphical documentation, including the watercolors, was carried out by D. Mackenzie (Momigliano, 1999), that was an archaeologist, second in charge after A. J. Evans, head of the mission. In this case he demonstrated a more straight and scientific method of depicting artistic features (Mackenzie, 1906).

Furthermore, the fact that the documentation of Kamares vessels from Knossos dated back to 1921-1935 (Evans, 1921) while that of Phaistos was performed in 1935-1951 and 1976 (Pernier, 1935), (Pernier, Banti, 1935), (Levi, 1976), it determined different choices in the reproduction strategies, like different line thickness or colors, due to the changing taste of the time (Figure6). Finally, another misleading problem in the interpretation of the Kamares pottery from Knossos come also from the reconstructive drawings, where in many cases the hypothesis of the missing part of a motif or of a decorative outline was denied by the subsequent findings. 
Figure 6. Watercolors of Kamares style pottery and potsherds: (a) Phaistos 1935 drawings by E. Stefani (Pernier, 1935); (b) Phaistos 1976 drawings by R. Oliva (Levi, 1976); (c) Knossos 1935 drawings by D. Mackenzie (Mackenzie, 1906)

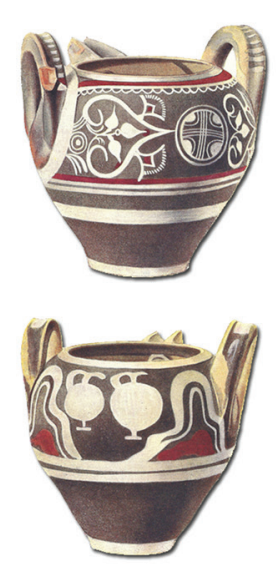

(a)

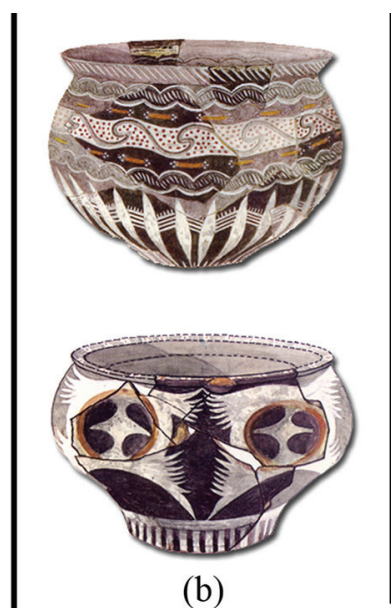

(b)

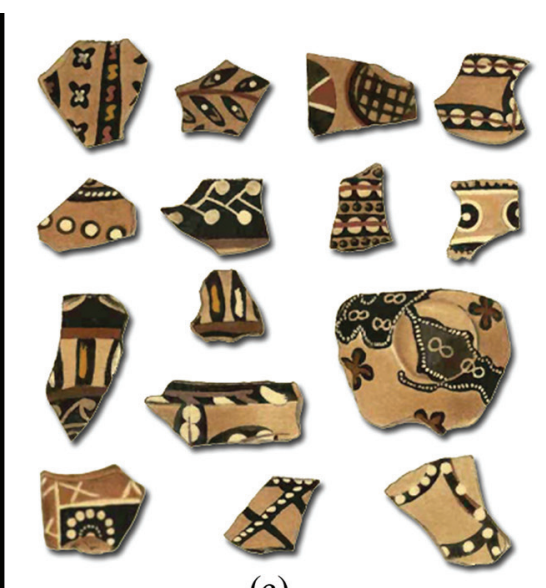

(c)

In this paper the results achieved working basically on the visual archive provided by Walberg's corpora (Walberg, 1983), (Walberg, 1987) will be discussed.

\section{KAMARES SHAPE ANALYSIS: SYSTEM OVERVIEW}

The automated system that we are building to assist the archaeologist in assessing similarities among the Kamares findings follows a general pipeline that could be easily adapted to other similar case studies. As in most Computer Vision applications the idea is to go from the raw data (pixels) to a symbolic representation of the image content. These information may, in turn, be fed to an intelligent system that assists the experts in formulating and checking working hypotheses about the scenes captured in the original images.

More specifically for the present case study the system starts with digitalized format of a pictorial representation of a vase or of a sherd. Figure 7 shows the successive steps performed. The digital data are the input of a contour extraction algorithm. Please, notice that in this way we are disregarding any $3 \mathrm{D}$ information: perspective deformation, and occlusions together with noise and artifacts due to low resolution are simply ignored at this stage.

Contour extraction produces a collection of contours: some of them are not relevant for our application and should be filtered out (Gonzalez, Woods, 2006), (Gonzalez, Woods, Eddins, 2009), (Moore, 1968). We choose to ask the interventa- 


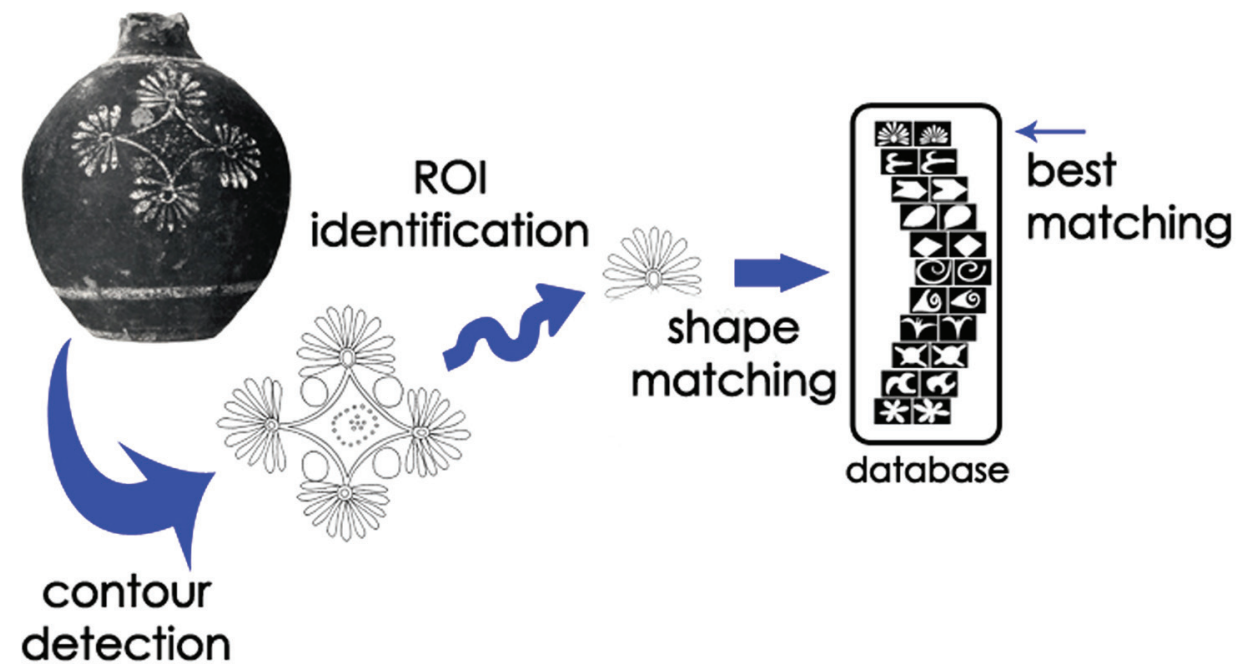

tion of the expert for this Region Of Interest (ROI) identification task because of the occurrence of cases like those reported in Figure 8. In the Figure several occurrences of the "raquet" decorative pattern are shown. It is clear that the isolation of this complex motif is the product of an informed semantic choice and that this expert choice is not easily mimicked with a fully automatic approach.

ROI identification most commonly reduces the set of contour shapes to examine. Even so Kamares decorative motifs are reduced by this step into a smaller but yet complex set of elementary shapes. Figure 9 shows several instances of the pattern that are obtained after ROI selection. Observe that in some case it is more convenient

Figure 8. Examples of occurrences of the "raquet" motif in different vases

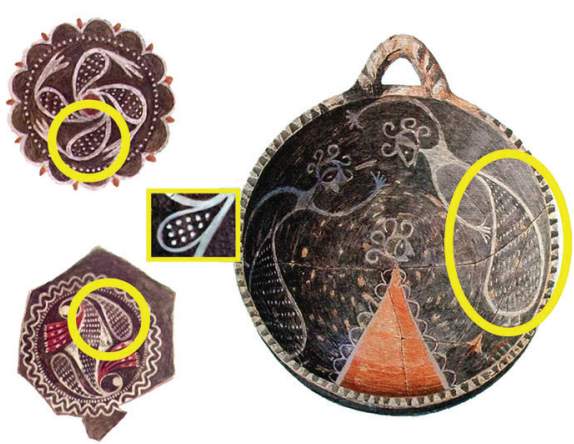

(a)

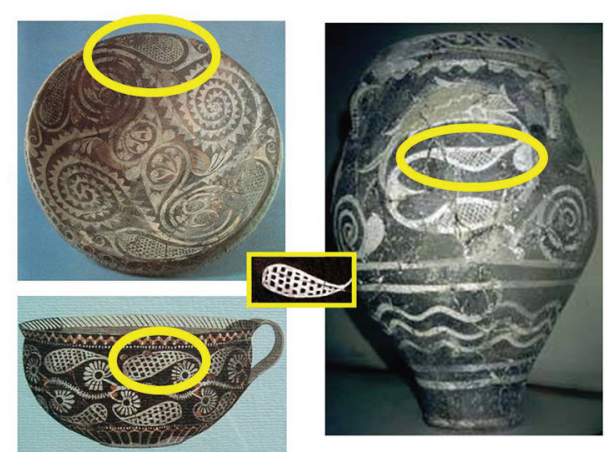

(b) 
to refer to the "flattened" version of the motifs whenever these, manually produced by an expert draftsman, are available. These lucky cases reduce the problems due to $3 \mathrm{D}$ distortion and occlusion but introduce in the pipeline the bias of the draftsman.

The proposed system deals with simpler shapes one at each time. They are analyzed by the shape analyzer and reduced to a set of numerical and geometrical invariants. These invariants are the final features that the matching module of the system considers for search and similarity retrieval into a reference shape database.

The reason to deal with the elementary simpler shapes instead that with the set of contours in a ROI has the following motivations:

- Algorithms that deal with simple contours and extract suitable invariants from them are much better understood and robust at the actual state of art;

- A ROI represents a complex figurative "proposition" composed of simpler elementary shapes. Unfortunately, the "order of reading" of such complex visual structures is not linear. Indeed it is not easy to "read" those drawing in a canonical un-ambiguous way.

\section{Shape Similarity Measures}

To apply the technique presented in this paper is necessary a contour line representation of the motifs. Hence, a decorative pattern is previously translated into a digital sequence of consecutive points on a raster plane.

Several issues about resolution and standardization of these rasterized contours arise. Going from digital images to digital shapes is far from being an easy task: different media (water colors, photos and hand drawn schematic lines) require different methods of contour extraction and present different algorithmic challenges. Once the pictorial data have been translated into a more abstract "contour shape"

Figure 9. Kamares potteries and relative decorations
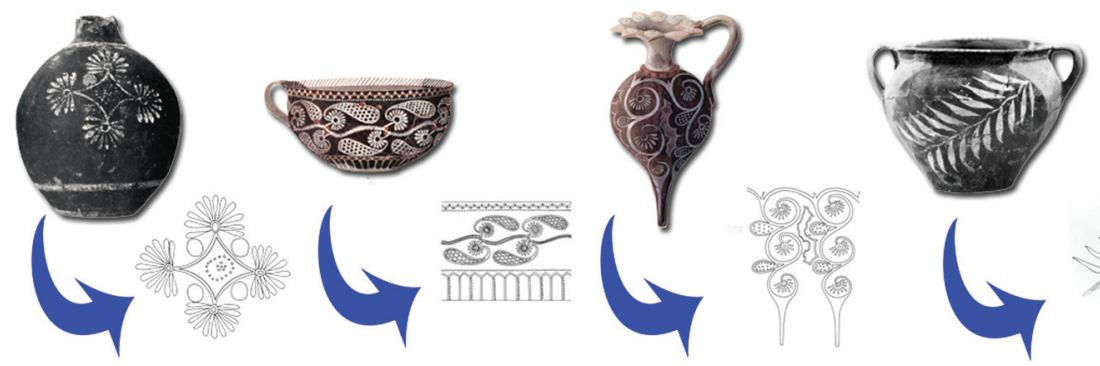
Figure 10. (a) Figure interpretation by the archaeologist. (b) Figure interpretation by the computer scientist. The same color indicates the same symbol.

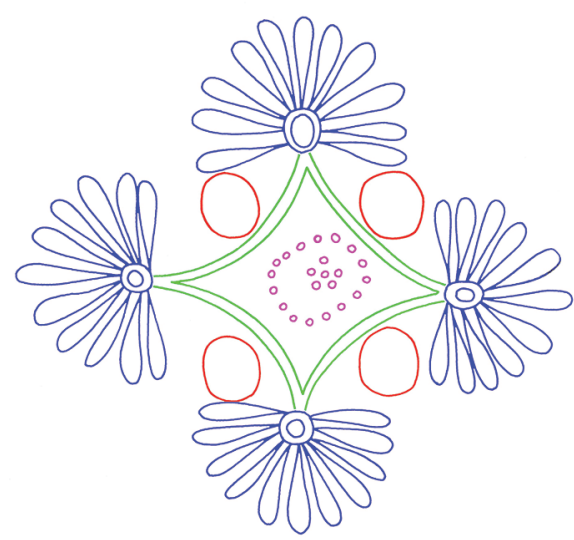

(a)

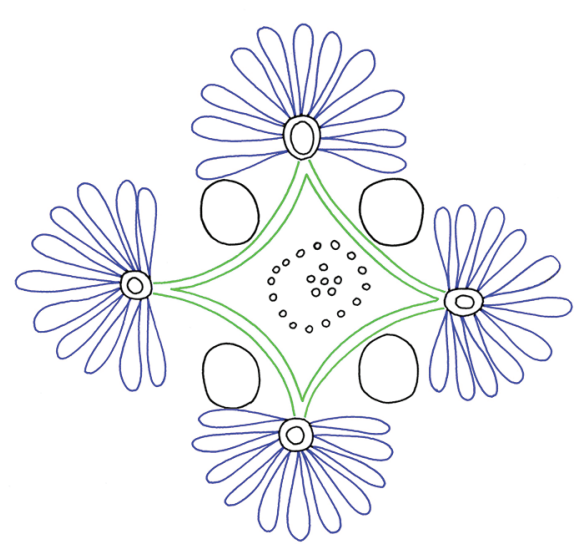

(b)

representation it is possible to apply to them the shape similarity measures presented below. The idea is to present the shape database with a "query" which represents a decorative element that the archaeologist wishes to recognize. The answer to the query is an estimate of the similarity distance between the query shape and the shapes in the database. In this chapter we present different shape similarity algorithms: contour flexibility technique (Xu, Liu, Tang, 2008), Shape Context (Belongie, Malik, Puzicha, 2002), logo recognition into document images (Shen, Jin,Chang, Wu, 2005), Procrustes methods (McNeill, Vijayakumar, 2006), and Circular Blurred Shape Model descriptor (Escalera, Fornes, Pujol, Escudero, Radeva, 2010).

Notice that even if the published ideas in shape recognition are overabundant in the present scientific literature most of them are relative to complex vision tasks where one wishes to recognize real objects that move and deform within a complex real environment. This is not the case for our application where the most common deformations observed are affine distortions, noise, irregularities, lacunae, and non linear scaling.

\section{Shape Matching using Shape Context}

Belongie et al. in 2002 propose a correspondence-based shape matching method using shape contexts (Belongie et al., 2002). Shape matching using shape contexts is an improvement to traditional Hausdorff distance based methods. It extracts a 
global feature, called shape context, for each corresponding point. The matching between corresponding points is then the matching between the context features. In this approach an object is treated as point set and it is assumed that the shape of the object can be captured by a finite subset of its points, for example sampled uniformly from the external contour. These points do not need to be key-points such as inflection points, cusps, extrema of curvature, etc., and can be derived from edge pixels found by a simple edge detector, whose output is a set $P$ of $n$ points $P=\left\{p_{1}\right.$, $\left.p_{2}, \ldots, p_{\mathrm{n}}\right\}, p_{\mathrm{i}} \in \mathrm{R}^{2}$.

The shape context considers the set of $n-1$ vectors originating from a point to all other sample points on a shape, which expresses the configuration of the whole shape with respect to the reference point. Since shapes may vary from an instance to another in the same category, the full set of vectors is too detailed and does not guarantee robustness. The distribution over relative position is a more robust and compact description, hence for each point $p_{\mathrm{i}}$ on the shape, a coarse histogram $h_{\mathrm{i}}$ of the relative $n-1$ coordinates is computed:

$$
h_{i}(k)=\#\left\{q \neq p_{i}:\left(q-p_{i}\right) \in \operatorname{bin}(k)\right\}
$$

The length $r$ and orientation $\theta$ of the vectors $\left(q-p_{\mathrm{i}}\right)$ are quantized to create a histogram map which is the shape context used to represent the point $p_{\mathrm{i}}$. To make the histogram more sensitive to the location of nearby points than to the location of points farther away, these vectors are mapped into a log-polar space representation (Figure11). Shape contexts are distribution represented as histograms: it is hence possible to use the $\chi^{2}$ test statistic to define the cost of matching two points. Consider two shapes $P$ and $Q$. A point $p_{\mathrm{i}}$ on the first shape and a point $q_{\mathrm{j}}$ on the second shape, $C_{\mathrm{ij}}=C\left(p_{\mathrm{i}}, q_{\mathrm{j}}\right)$ denotes the cost of matching the two points in exam, where $C\left(p_{i}, q_{j}\right)=1 / 2 \sum_{k=1}^{K} \frac{\left[h_{i}(k)-h_{j}(k)\right]^{2}}{h_{i}(k)+h_{j}(k)}$ and $h_{\mathrm{i}}(k), h_{\mathrm{j}}(k)$ denote the $K$ - bin normalized histogram respectively at $p_{\mathrm{i}}$ and $q_{\mathrm{j}}$. Given the set of costs $C_{\mathrm{ij}}$ between all pairs of points $p_{\mathrm{i}}$ of $P$ and $q_{\mathrm{j}}$ of $Q$, the total cost of matching $H(\pi)=\sum_{i} C\left(p_{i}, q_{\pi(i)}\right)$ has to be minimized, with the constraint of one-to-one matching. The matching of two shapes then is done by matching two context maps of the shapes, which is a matrixbased matching. It minimizes the total cost of matching between one context matrix and all the permutations of another context matrix. To reduce the matching overhead, the shortest augmenting path algorithm for the matrix matching is used. When the number of sample points is not equal on the two shapes, the matching is done adding dummy nodes to the smaller point set, with a constant matching cost of $\varepsilon_{i}$. Since all measures are taken with respect to points on the object, invariance to translation 
Figure 11. The log polar maps of the contest of two different points in the contour of a shape
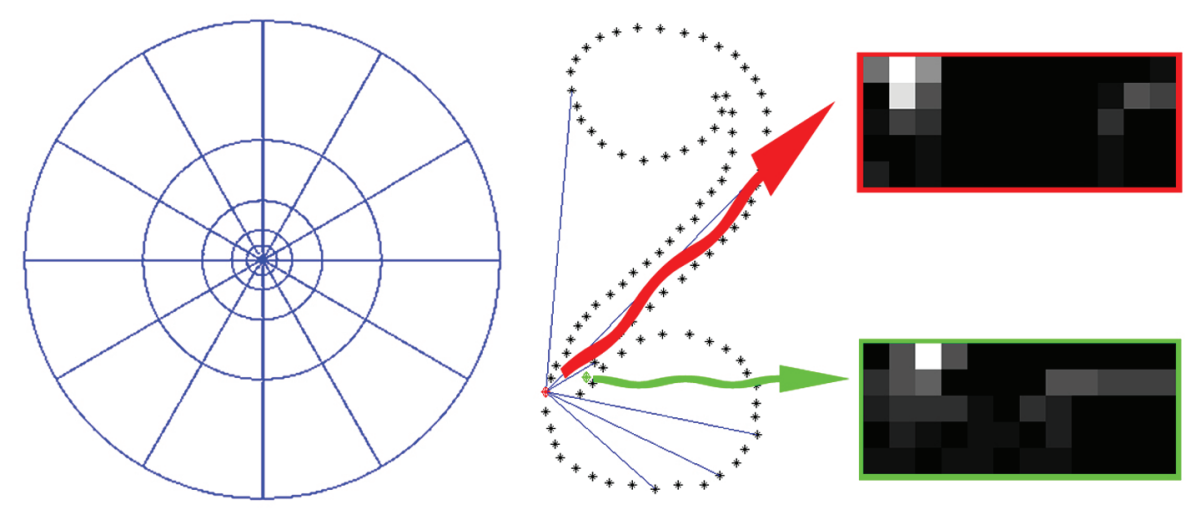

is intrinsic to the definition of the descriptor. The use of histograms makes shape context insensitive to small deformations, non linear transformation occlusions and presence of outliers. Additional robustness to outlier can be added not allowing points labeled as outlier to contribute to any histogram. The above definition of shape context is not invariant to rotation, which can be obtained using a relative frame instead of the absolute frame described, e.g., treating the tangent vector at each point as the positive $x$-axis.

\section{Hierarchical Procrustes Matching}

Hierarchical Procrustes Matching (HPM) (McNeill, Vijayakumar, 2006) is a segment-based shape matching algorithm that approximates the perceptual similarity between two shapes by matching progressively smaller boundary segments. The denomination makes reference to a mythological character that used to stretch or cut his guests to make them fit to his bed. Corresponding regions of shapes often appear at slightly different scales and positions and matching algorithms must be flexible to adjust these variations. Simple global point methods (McNeill, Vijayakumar, 2005), (Zhang, Zhang, Krim, Walter, 2003) and non linear approaches (Bookstein,1997), (Sebastian, Klein, Kimia, 2003) suffer from presence of smooth deformation and may produce a large discrepancy between a perceived difference and its numerical estimation. HPM avoids this by matching in a global to local direction. Longer segments that have been matched provide initial matches for the shorter segments, which can then slide and stretch/contract in order to find the best matches at this smaller scale. 
Given two objects represented by n 2 D points: $U=\left(U_{1}, \ldots, U_{N}\right)^{N}$ and $V=\left(V_{1}\right.$, $\left.\ldots, V_{N}\right)^{N} \in \mathrm{R}^{N \times 2}$ this approach normalizes the boundary length of the polygon associated with $V$ and then transforms $U$ to match $V$. Each point is represented as a complex number $V_{n}=\left(x_{n}, y_{n}\right) \rightarrow x_{n}+i y_{n}=w_{n} \in \mathrm{C}$. Then, $V \rightarrow w$ and $U \rightarrow z \in \mathrm{C}^{N}$. After centering $w$ and $z$ so that $\sum_{n=1}^{N} w_{n}=\sum_{n=1}^{N} z_{n}=0$, the Procrustes Distances (PD) between $U$ and $V$ is given by

$d_{p}(U, V)=\left(w^{*} w-\left(w^{*} z z^{*} w\right) / z^{*} z\right) / N$

In order to compute HPM is necessary to compute the Global Procrustes Matching (GPM) that find the best correspondence between $U$ and $V$ with the smallest PD among the $N$ valid correspondences that respect the cyclic order of the indices. This initial correspondence is in turn refined using a vector that is one half of $V$ and finding the smallest PD with each "half" of $U$. The matched segments at this length provide initial estimates for matching at the successive scale that can be performed recursively. The shape similarity of $U$ and $V$ is the weighted sum of scores at each length, where the weight is related to the segments length. Let $S_{l}$ denote the sum of PDs over the matched segments at a fixed length $l$. The asymmetric similarity of $U$ and $V$ is given by

$$
d_{F}(U, V) \equiv w_{100} d_{p}(U, V)+\sum_{l=50,25,12.5} w_{l} s_{l}
$$

where the $w_{l}$ are constant weights, 100 is the boundary length, and 50, 25 and 12.5 are length of segmentswhen we divide the boundary. Since $d_{F}(U, V) \neq d_{F}(V, U)$, the symmetric similarity is given by

$$
D_{F}(U, V) \equiv d_{F}(U, V)+d_{F}(V, U)
$$

In order to consider the average confidence with which each segment match is selected, the PDs are normalized by making the average PD equal to 1, and then apply HPM as normal. This is also known in literature as Normalized Procrustes Distances. 


\section{Shape Matching by Contour Flexibility}

It this Section we briefly report the main ideas about contour flexibility. The reader is urged to find the details of this algorithm in (Xu, Liu, Tang, 2008). Vision science has developed as an interdisciplinary research field, frequently involving concepts and tools from computer science, image processing, biology, psychology and cognitive science, for example the importance of high curvature points in shape perception has been already described in (Atteave, 1954) and (Biderman, 1985). From this theories the important features for shape recognition come from parts of the object where changes occur (e.g., corners). A recent descriptor, named contour flexibility (Xu, Liu, Tang, 2008), depicts the deformable potential at each point along a closed curve, extracting both global and local features, with proper trade-off between them. It can be observed that in most cases articulated high flexible parts correspond to high curvature points. Let $\ell$ be a closed simple contour, surrounding a bounded domain $D$. For a point $\mathrm{p}$ on a contour $\ell$ and a given radius $r$, the contour flexibility is defined as $\omega(p, r)=\min \left(\omega_{+}(p, r), \omega_{-}(p, r)\right)$, where $\omega_{+}$is the interior flexibility and $\omega_{\text {_ }}$ is the exterior flexibility:

$$
\begin{aligned}
\omega_{+}(p, r) & =\frac{\int_{C_{p, r}^{+}} k_{+}(x) d x}{\int_{C_{p, r}^{+}} d x} \\
\omega_{-}(p, r) & =\frac{\int_{C_{p, r}^{-}} k_{-}(x) d x}{\int_{C_{p, r}^{-}} d x}
\end{aligned}
$$

$C_{p, r}^{+}$and $C_{p, r}^{-}$respectively are the connected components containing $p$ in the sets $\{x \in D:\|x-p\| \leq r\}$ and $\left\{x \in \mathrm{R}^{2}-D:\|x-p\| \leq r\right\} . \mathrm{k}_{+}$and $\mathrm{k}_{-}$are two function of distance transform on $\mathrm{R}^{2}$,

$$
k_{+}(x)=d\left(x, \Re^{2} \backslash D\right) \text { and } k_{-}(x)=d(x, D), \quad x \in \Re^{2}
$$

$D(\cdot, \cdot)$ is the minimum Euclidean distance between two sets. The radius $r$ is called bendable size and should be tuned taking into account the width of the limb-like parts of an object. The bendable size $r$ and the contour flexibility are proportional to the scale of the contour but invariant to translation, rotation and the choice of starting point for the parameterization of the contour. Let 
$z(t)=(x(t), y(t)) ; 0 \leq t<1$

be the arc-length parameterization of a contour $\ell$. Since it is difficult to match flexible parts of two contours (e.g., using uniform sampling of the contour), a better strategy is to give large weights to inflexible landmarks and smaller weights to more flexible landmarks, using more samples on the segments of a contour which are more inflexible. Let

$$
\gamma(t)=\frac{\sum_{0}^{t} \omega(z(u), r) d u}{\sum_{0}^{1} \omega(z(u), r) d u}
$$

the optimal sampling can be obtained with sampling speed $d \gamma / d t$. Considering each landmark a complex number $z^{k}=x^{k}+j y^{k}$, the contour can be treated as a complex vector $\Phi=\left(z_{1}, z_{2}, \ldots, z_{n}\right)^{T}$ and the Procrustean Distance between two sequences can be used for global matching of $\bar{\Phi}_{1}$ and $\bar{\Phi}_{2}$ :

$$
\hat{d}\left(\bar{\Phi}_{1}, \bar{\Phi}_{2}\right)=\cos ^{-1}\left(\max _{\mathrm{S} \in N}\left|\left\langle\bar{\Phi}_{1}, \sigma^{s}\left(\bar{\Phi}_{2}\right)\right\rangle\right|\right)
$$

where $\sigma^{s}\left(\bar{\Phi}_{2}\right)$ is a cyclic permutation of $\bar{\Phi}_{2}$, to achieve independence from the starting point and " \langle\rangle " denotes the inner product of two complex vectors. The matching score between the two shapes represented by $\bar{\Phi}_{1}$ and $\bar{\Phi}_{2}$ is determined by

$$
M_{d}=\frac{\alpha}{\pi} \hat{d}\left(\bar{\Phi}_{1}, \bar{\Phi}_{2}\right)+D\left(\Omega_{1}, \Omega_{2}\right)
$$

Where $D(\cdot, \cdot)$ is the warping distance between the two sequences of the contour flexibility values extracted from the contours; $\Omega_{1}, \Omega_{2}$ are the sequences of the values of the contour flexibility at each landmark of uniformly sampled sequences, starting form the leading landmark $\hat{s}$ found by (1.10), $\alpha$ weighting factor, $\pi$ normalization factor. 


\section{Shape Matching by Contour Flexibility}

Circular Blurred Shape Model is a recent rotationally invariant descriptor, which makes use of a correlogram structure to capture spatial arrangement of object parts, shared among regions defined by circles and sections (Escalera et al., 2010). The correlogram structure can be defined as follows: given a number of circles $C$, number of sections $S$, and an image region $I$, a correlogram $B=\left\{b_{\{1,1\}}, \ldots, b_{\{\mathrm{C}, \mathrm{S}\}}\right\}$ is a radial distribution of sub-regions of the image. In each region $b$ of the correlogram the centroid $b^{*}$ can be located by its coordinates and used as reference point. The regions around $b$ define the neighborhood of $b$, and the number of neighbors depends on the spatial location $b$ (e.g. inner circle, middle circle or extern circle).

The descriptor makes use of the information obtained from the contour of the object, which can be extracted for example by means of an edge detector. Every point $x$ in the contour map is taken into account, calculating first the distance to the corresponding centroid of the region in which $x$ lies, then the distances to the centroids of neighbor regions. The inverse of these distances are computed and normalized by the sum of total distances and the obtained values are then added to the corresponding positions of the descriptor vector $n$, which as dimension $C \times S$. As in the case of shape context, the use of histograms makes description tolerant to irregular deformations. As for the complexity, for a map of $k$ relevant contour points, the computation of the descriptor requires $O(k)$ simple operations. Parameters $C$ and $S$ defines the degree of spatial information taken into account in the description process (i.e., as the number of regions increase the description becomes more local) and should be tuned for each particular application. See also Figure 12. To obtain a rotationally invariant descriptor a second step needs to be included. The main diagonal $G_{\mathrm{i}}$ of correlogram $B$, with the highest density, is searched. This diagonal is then used as a reference to rotate the descriptor. The orientation of the rotational process, so that $G_{\mathrm{i}}$ is aligned with the $x$-axis, is that corresponding to

Figure 12. Circular Blurred Shape Models of the same shape with different choices of the parameters $C$ and $S$.

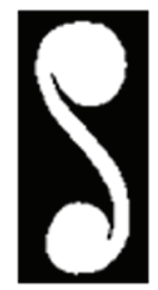

a

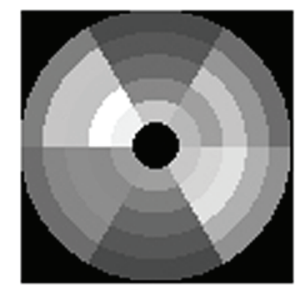

b

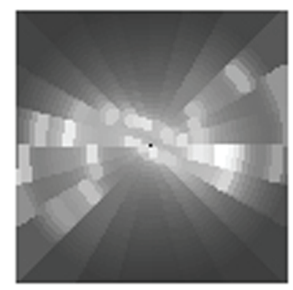

C

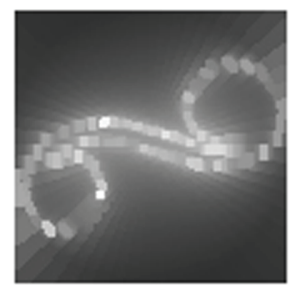

d 
the highest description density at both sides of $G_{\mathrm{i}}$. Once obtained the rotationally invariant CBSM descriptor, it can be used to design a symbol spotting methodology (e.g., using Adaboost to distinguish between foreground and background) or a multi-class classifier by embedding binary classifiers, for instance using Adaboost to define a classifier based on the features that best discriminate one class against another, combining then binary classifiers in a Error Correcting Code Framework.

\section{EXPERIMENTAL RESULTS}

To test our system we have built an experimental image database. The database has been created as follows. First of all, a selection of images from (Walberg, 1978) have been inserted in the collection. A total of 36 motifs have been chosen. The selection of these motifs among the much larger catalogue in (Walberg, 1978) has been done randomly, although in this initial stage of our research we choose to sort out the most complex motifs. Instances of these chosen patterns show a high-class variability in terms of scale, rotation, rigid and elastic deformations when observed in the pictorial reference corpus. Some of the shapes exhibits a low inter-class variability especially for simpler shapes. This explains for example some resulting ambiguities of the classification. The images selected are in a way the "canonical" reference for each of the decorative motifs to recognize. To use as a reference only the clean and canonical drawing prepared by an expert is too demanding for any shape recognition algorithm. Following a general praxis in the shape recognition community we hence enriched the database with three variations for each decorative motif. These variations have been artificially obtained applying the following transformations: rotation in clockwise sense of about 33 degrees, perspective camera distortion where camera orientation has been assigned at random and random warping. Examples of the shape included in the database are reported in Figure 13, an example of variations over a given shape is reported in Figure 14.

Eventually the database is made of 144 shapes. Those shapes have been stored in binary images of $500 \times 500$ pixels. Those images have been processed for contour extraction in Matlab (Lorensen, Cline, 1987), (Maple, 2003). The resulting contours have been resampled and for each shape a vector of 100 points have been obtained. Using a higher sampling rate does not improve the performance of the system, perhaps because a denser sampling of a contour tends to preserve some of the noise of the original image making recognition and matching a harder task. The down-sampling has been performed in two different ways, and the results of both selections have been stored in the reference database. The first way to sample 100 points from a closed contour is to compute 100 equally spaced pints along the 
Figure 13.
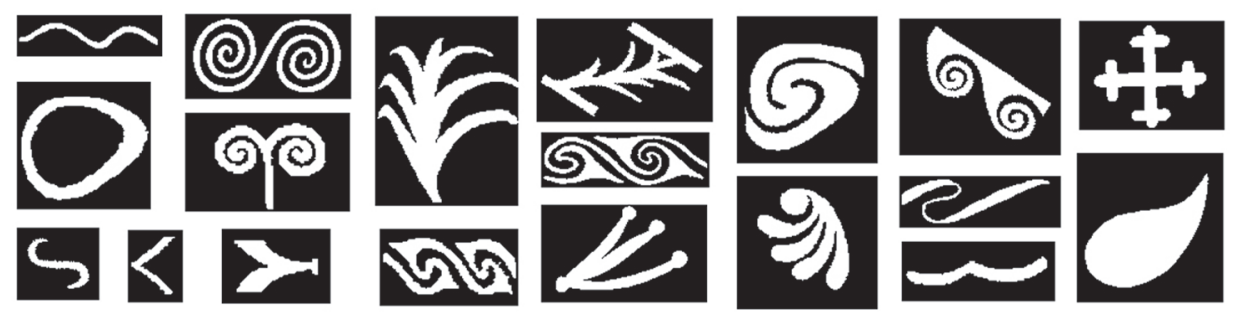

Figure 14.
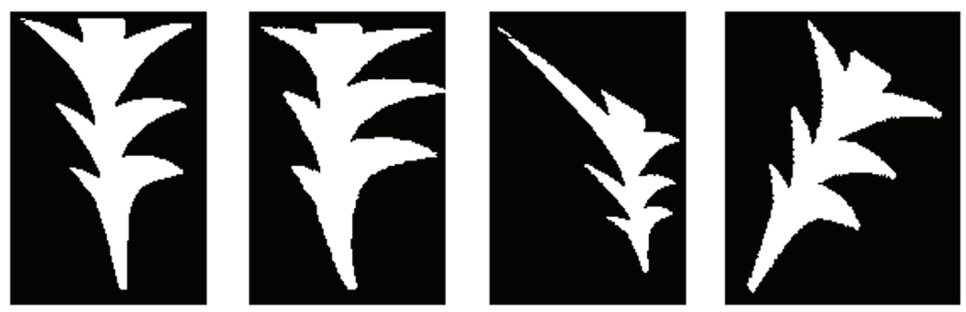

contour. Notice that the sample points are obtained with cubic interpolations of close by points on the contour. This uniform sampling procedure is applied to be able to compute CBSM distance and to estimate local contour flexibility values. A second non-uniform sampling has been also performed. This second sampling takes 100 points according to contour flexibility values: more points are allocated in the most flexible segments of the shape. This non-uniform sampling procedure is applied to be able to compute contour flexibility distance. Contour flexibility value in each of the sampled point is also stored. In summary our system refers to this collection of $144 \times 2$ point vectors and 144 contour flexibility values to compute similarity distances of queries from the database. Observe that this mathematical representation is quite compact and easily allows the scaling up of the system up to thousands of shapes.

We choose to check the performance only of the CSBM algorithm and of the Contour Flexibility approach. Indeed CSBM is a refinement of the initial Belongie's proposal and it is safe to assume that it will perform better. A similar reasoning justifies the choice of Contour Flexibility over Hierarchical Procrustes Distance for our tests. 
The experiments have been carried out as follows. Each shape in the database has been, in turn, considered as a query and the best matching shapes (other than the query shapes itself) have been considered as the results of the retrieval operation. In Table 1 we reported the results obtained with this set of experiments. In particular the first column of the Table indicates the percentage of total queries when the algorithms found, within the four most similar shapes, the query shape itself and the three variations of it in the database. The second column in the table indicates the percentage of total queries when the algorithms found, within the three most similar shapes (other than the query shape itself) the majority of shapes of the correct class.

Table 1. Performance of the shape matching procedures

\begin{tabular}{|l|c|c|}
\hline & $\begin{array}{c}\text { \% of perfect matches within } \\
\text { the four closest shapes }\end{array}$ & $\begin{array}{c}\text { \% of majority of correct } \\
\text { matches within the four } \\
\text { closest shapes }\end{array}$ \\
\hline Circular Blurred Shape Model (CBSM) & $48 \%$ & $72 \%$ \\
Contour Flexibility (CF) & $75 \%$ & $99 \%$ \\
\hline
\end{tabular}

Figure 15. Instance of false positives produced by the system, (a) CF; (b) CBSM
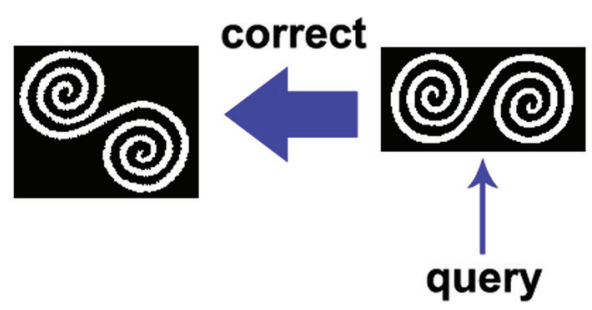

wrong
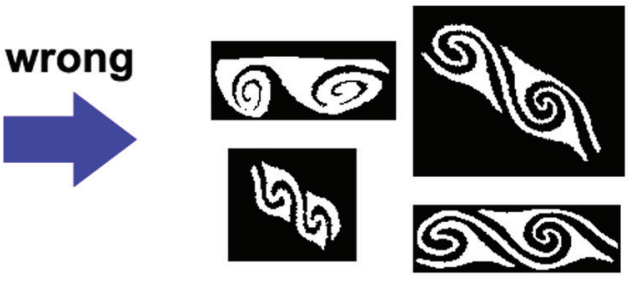

(a)
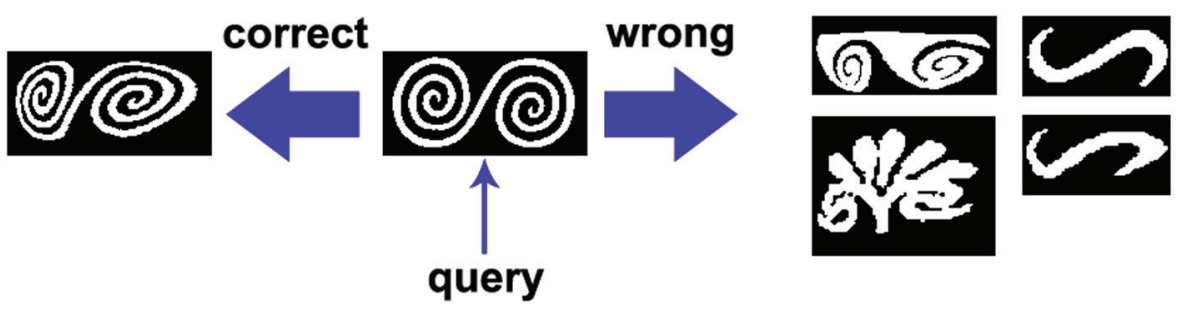

(b) 
A closer observation of the results reveals that most of the errors are "near misses". An example of these is given in Figure 15.

The results are very encouraging, although the system is far from being error free it provides a valuable help to the expert. When trying to match a novel query an archaeologist, in almost all of the cases, has to look only at a very small (4 to 6) reference shapes provided as an answer from the system. This greatly reduces the time that he should otherwise spend to look among a larger set of candidate reference patterns. On the other hand at this stage of development of the system the human expert intervention is still necessary.

\section{Real World Examples}

To further support our claim about the robustness and the usefulness of our approach we present two real world examples. The queries and the results are shown in Figure 16. The application of the algorithm for the automatic classification of the Kamares style pottery revealed itself particularly efficient for the immediate discrimination of the motifs on the chronological and geographical scales. Basing upon the Walberg's corpora it's possible to offer two examples of the procedure working. In the first case in Figure16(a), we have a potsherd with a spiral type motif partially preserved. From the image the algorithm automatically extracts the contour and checking for matches in the available database it suggests three hypothesis of interpretation, the simple j-spiral (Walberg 2.i.1), the running j-spiral (Walberg 2.i.2), even possibly deformed, and the $\mathrm{j}$-spiral with filled angle (Walberg 6.2), again with a certain degree of alteration. The immediate restriction of the range of possible motifs gives to the archaeologists the chance to easily check the available data on the geographical and chronological distribution of the motifs themselves and obtain confirmation for their initial hypothesis. In fact, while the simple J- spirals has a wide diffusion in both Provincial and Palatial areas and in the four chronological phases, the j-spiral with filled angle is present in the Palatial area just in the phase 3 and in the Provincial district exclusively in the phases 2 and 3 and just in the East and East-Central Crete (in (Walberg, 1983) pp. 38-39, 41, pll. 28, 31; (Walberg, 1987), pp. 48-49, 51, 180, 183). Again in the second example in Figure16(b), in the potsherd is visible a series of teardrops motifs. After the extraction of the contour, the algorithm suggests two different matches, the spiral derivatives (Walberg 8.33), both the original motif and its altered versions, and the petaloid loops (Walberg 12.i.1). Also in this case, the two motifs have exclusive distribution features. The spiral derivatives are present in the Palatial area in phases 2-4 and are absent in phase 1, while in the Provincial district they occur in all the chronological phases but restricted just to East, EastCentral and Central Crete. The petaloid loops have same distribution in the Palaces as the above mentioned derivatives but in the Provincial areas they are exclusive of 
Figure 16. Examples of real queries to the database
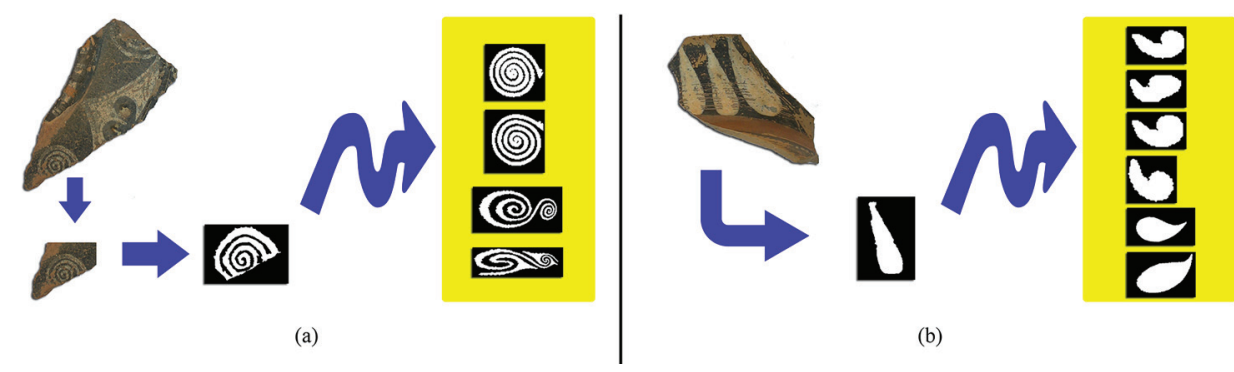

(b)

phase 1 and 3 also having the same geographical diffusion ((Walberg, 1983), pp. 43-44, 48-49, pll. 35, 41; (Walberg, 1987), pp. 52-53, 57-58, 183, 188).

\section{CONCLUSION AND FUTURE WORK}

In this chapter a pipeline to automatically classify simple Kamares decoration has been presented. In particular, we focused on the shape matching of the pipeline showing how contour flexibility together with Procustean distance, circular blurred shape model or shape context may solve this problem. The proposed system will be of great support in automatically assisting the archaeologists in classification operations according time and place of production of Kamares pottery fragments.

This first example of application of Computer Vision and Pattern Recognition techniques to a specific topic of the prehistoric archaeology as the Minoan Kamares pottery opened a completely new field of investigation that in the future can deeply change the approaching of the scholars to iconographical problems. The satisfactory results obtained with the classification of single motifs, even deformed by the preservation of the specimens or altered by the chronological and geographical variability of the production and by the creativity of the Minoan artisans, let expect the extension of this technique also to the decorative syntax of the Kamares style, in order to allow the archaeologist to look forward for the long waited exhaustive study of this so significant feature of the Mediterranean prehistory.

\section{ACKNOWLEDGMENT}

The authors wish to thank Professors V. La Rosa and F. Carinci for their precious support and the many useful advices that they have provided during this research. 


\section{REFERENCES}

Attneave, F. (1954). Some informational aspects of visual perception. Psychological Review, 61, 183-193. doi:10.1037/h0054663

Belongie, S., Malik, J., \& Puzicha, J. (2002). Shape Matching and Object Recognition Using Shape Contexts. IEEE Transactions on Pattern Analysis and Machine Intelligence, 24, 509-522. doi:http://doi.ieeecomputersociety.org/10.1109/34.993558

Biderman, I. (1985). Human image understanding: Recent research and a theory. Computer Vision Graphics and Image Processing, 32, 29-73. doi:10.1016/0734189X(85)90002-7

Bookstein, F. (1997). Landmark methods for forms without landmarks: morphometrics of group differences in outline shape. Medical Image Analysis, 1(3), 225-243. doi:10.1016/S1361-8415(97)85012-8

Carinci, F. (1997). Pottery workshops at Phaestos and Haghia Triada in the protopalatial period, in Aegaeum (Vol. 16). Center of Cretan Archaeology. (S.d.). Center of Cretan Archaeology. Retrieved July 31, 2010 from http://www.cac.unit.it

Dawkins, R. M., \& Laistner, M. (1912). The Excavation of the Kamares Cave in Crete. in Annual of the British School at Athens, 19, 1-34.

Day, P. M., \& Wilson, D. E. (1998). Consuming power. Kamares ware in protopalatial Knossos. Antiquity, 72, 350-358.

Escalera, S., Fornes, A., Pujol, O., Escudero, A., \& Radeva, P. I. (2009). Circular Blurred Shape Model for symbol spotting in documents. In ICIP09 (pp. 2005-2008).

Evans, A. (1921). The Palace of Minos at Knossos. London.

Farinella, G. M., Stanco, F., \& Tanasi, D. (2008). Digital-Kamares: un sistema automatizzato per riconoscere e classificare la ceramica minoica di stile Kamares. In AIAr 2008, V Congresso Nazionale di Archeometria, Scienza e Beni Culturali (pp. 547-556).

Gallo, G., Stanco, F., \& Tanasi, D. (in press). Riconoscimento automatico di motivi decorativi nelle ceramiche minoiche di stile Kamares da Festós. In 13 a Giornata di Archeometria della Ceramica: Nuove metodologie analitiche e di trattazione dei dati nello studio della ceramica in archeologia ed in architettura.

Gonzalez, R. C., \& Woods, R. E. (2006). Digital Image Processing (3rd ed.). Upper Saddle River, NJ: Prentice-Hall, Inc. 
Gonzalez, R. C., Woods, R. E., \& Eddins, S. L. (2009). Digital Image Processing Using MATLAB. Upper Saddle River, NJ: Gatesmark Publishing.

Guarnera, G. C., Stanco, F., Tanasi, D., \& Gallo, G. (2010). Classification of decorative patterns in Kamares pottery. In In proceedings of SCCG $26 y$ h Spring Conference on Computer Graphics (pp. 20-23). Comenius University, Bratislava.

IPLab. (S.d.). Archeomatica Project. Retrived July 31, 2010 from http://www. archeomatica.unict.it

Knappett, C. (1999). Tradition and innovation in pottery forming technology. Wheelthrowing at Middle Minoan Knossos. BSA, 94, 101-129.

Levi, D. (1976). Festós e la Civiltá Minoica I, Roma.

Levi, D., \& Carinci, F. (1988). Festós e la Civiltá Minoica.

Lorensen, W. E., \& Cline, H. E. (1987). Marching cubes: A high resolution 3D surface construction algorithm. In SIGGRAPH '87: Proceedings of the 14th annual conference on Computer graphics and interactive techniques (pp. 163-169). New York: ACM. doi:http://doi.acm.org/10.1145/37401.37422

Macgillivray, J.A. (1998). Knossos. Pottery groups of the Old Palace period. London.

Mackenzie, D. (1906). The Middle Minoan pottery of Knossos. Journal of Hellenic Studies.

Maple, C. (2003). Geometric design and space planning using the marching squares and marching cube algorithms. In Geometric Modeling and Graphics, 2003. Proceedings. 2003 International Conference on (pp. 90 - 95). doi:10.1109/ GMAG.2003.1219671

McNeill, G., \& Vijayakumar, S. (2005). 2D shape classification and retrieval. In IJCAI'05: Proceedings of the 19th international joint conference on Artificial intelligence (pp. 1483-1488). San Francisco: Morgan Kaufmann Publishers Inc.

McNeill, G., \& Vijayakumar, S. (2006). Hierarchical Procrustes Matching for Shape Retrieval. In CVPR '06: Proceedings of the 2006 IEEE Computer Society Conference on Computer Vision and Pattern Recognition (pp. 885-894). Washington, DC, USA: IEEE Computer Society. doi:http://dx.doi.org/10.1109/CVPR.2006.133

Momigliano, N. (1999). Duncan Mackenzie: a Cautious Canny Highlander and the Palace of Minos at Knossos. (Vol. 72). Bulletin of the Institute of Classical Studies, London. 
Moore, G. A. (1968). Automatic Scanning and Computer Processes for the Quantitative Analysis of Micrographs and Equivalent Subjects. In PPR68 (pp. 275-326).

Pernier, L. (1935). Il palazzo minoico di Festós.

Pernier, L., \& Banti, L. (1951). Il palazzo minoico di Festós.

Sebastian, T. B., Klein, P. N., \& Kimia, B. B. (2003). On Aligning Curves. IEEE Trans. Pattern Anal. Mach. Intell., 25(1), 116-125. doi:http://dx.doi.org/10.1109/ TPAMI.2003.1159951

Shen, D. F., Jin, L., Chang, H. T., \& Wu, H. H. (2005). Trademark Retrieval Based On Block Feature Index Code. In ICIP05 (pp. III: 177-180).

Stanco, F., Battiato, S., \& Gallo, G. (2011). Digital Imaging for Cultural Heritage Preservation. Analysis, Restoration ad Reconstruction of Ancient Artworks. CRC Press.

Walberg, G. (1978). The Kamares style. Overall effects (Vol. 10). Uppsala: Acta Universitatis Upsaliensis. Boreas.

Walberg, G. (1983). Provincial Middle Minoan pottery. Mainz.

Walberg, G. (1987). Kamares. A study of the character of Palatial Middle Minoan pottery. Göteborg.

Walberg, G. (2001). The role and individuality of Kamares ware. in Aegean Archeaology, 5, 9-18.

Xu, C., Liu, J., \& Tang, X. (2008). 2D Shape Matching by Contour Flexibility. IEEE Transactions on Pattern Analysis and Machine Intelligence, 31, 180-186. doi:http:// doi.ieeecomputersociety.org/10.1109/TPAMI.2008.199

Zhang, J., Zhang, X., Krim, H., \& Walter, G. G. (2003). Object representation and recognition in shape spaces. Pattern Recognition, 36(5), 1143-1154. doi:10.1016/ S0031-3203(02)00226-1

Zois, A. A. (1968). Der Kamares-Stil. Werden und Wesen (Diss.), Tübingen. 